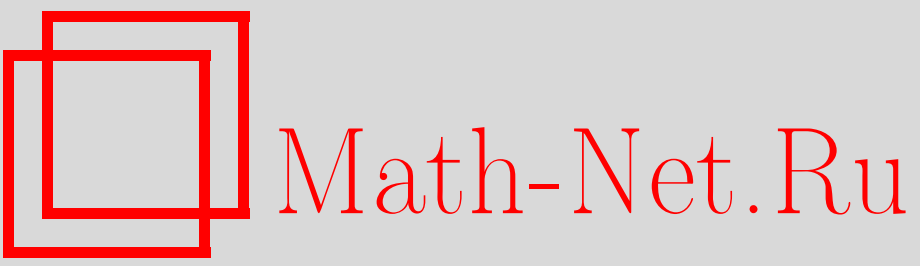

В. Д. Гордевский, Приближенное двухпотоковое решение уравнения Больцмана, ТМФ, 1998, том 114, номер 1, 126-136

DOI: https://doi.org/10.4213/tmf833

Использование Общероссийского математического портала Math-Net.Ru подразумевает, что вы прочитали и согласны с пользовательским соглашением

http://www.mathnet.ru/rus/agreement

Параметры загрузки:

IP : 3.85 .5 .30

26 апреля 2023 г., 05:02:42 
ТЕОРЕТИЧЕСКАЯ

И МАТЕМАТИЧЕСКАЯ

ФИЗИКА

Том 114, № 1

январь, 1998

(C) 1998 r.

В. Д. Гордевский*

\section{ПРИБЛИЖЕННОЕ ДВУХПОТОКОВОЕ РЕШЕНИЕ УРАВНЕНИЯ БОЛЬЦМАНА}

Построено явное приближенное решение трехмерного нелинейного уравнения Больцмана для твердых сфер. Оно имеет вид пространственно неоднородной линейной комбинации двух максвеллианов, отвечающих разным плотностям, температурам и массовым скоростям. Показано, что за счет выбора параметров, входящих в распределение, невязка между левой и правой частями уравнения в интегральной норме может быть сделана сколь угодно малой.

\section{1. ВВЕДЕНИЕ}

В исследованиях, посвященных кинетическому уравнению Больцмана [1], важное место занимает поиск точных решений этого уравнения. Хорошо известны частные решения в виде глобального и локального максвеллианов $[1,2]$. Больших успехов удалось достичь в частном случае максвелловских молекул [3-10]. Были получены решения (иногда лишь в виде формальных рядов) и для некоторых более обших случаев [11-14]. Однако для физически содержательных моделей взаимодействия, в частности для модели твердых (упругих) сфер, до сих пор неизвестно явное решение, отличное от максвеллиана. В связи с этим возникает интерес к поиску приближенных явных решений нелинейного уравнения Больцмана, удовлетворяюших ему лишь с произвольной степенью точности. Такие решения служат хорошей аппроксимацией некоторых точных решений, описываюших неравновесные состояния газа, и в силу своей простоты являются удобной моделью для истинных решений уравнения Больцмана, сильно отличаюшихся от максвеллианов. Заметим, что первые попытки в этом направлении были предприняты еше в послевоенные годы в связи с приближенным описанием ударной волны (распределение Тамма-Мотт-Смита [1, с. 414; 15]).

В работе [16] построено приближенное бимодальное решение трехмерного нелинейного уравнения Больцмана для твердых сфер. Оно имеет вид линейной комбинации двух глобальных максвеллианов с одинаковыми температурами и плотностями, но разными массовыми скоростями, и с коэффициентами специального вида, зависящими от пространственных координат $x$ и времени $t$ (в [16] оно названо "заворачиваюшим потоком"). В работах $[16,17]$ показано, что при стремлении температуры к нулю само решение

\footnotetext{
* Харьковский государственный университет, Харьков, Украина
} 
$f(t, v, x)$, а также левая и правая части уравнения Больцмана $D(f)$ и $Q(f, f)$ не выдерживают предельного перехода в пространстве $L$ с нормой

$$
\|g\|_{L}=\sup _{(x, t) \in R^{3} \times R^{1}} \int_{R^{3}}|g(t, v, x)| d v,
$$

однако норма разности между $D(f)$ и $Q(f, f)$ в $L$ имеет конечный предел. Этот предел точно вычислен в работе [17], причем показано, что он может быть сделан сколь угодно малым при больших числах Кнудсена и соответствуюшем выборе параметров, характеризующих распределение $f$. Это означает, что при "околосвободномолекулярном" течении газа возможны потоки, сколь угодно близкие к бимодальным. Отметим, что даже в смысле обобшенных функций распределение $f$ не стремится к максвеллиану в пределе низких температур, т.е. найденное приближенное решение не есть малое отклонение от состояния равновесия.

Целью настоящей работы является исследование поведения обобщенного бимодального распределения (назовем его двухпотоковым), в котором не только массовые скорости максвеллианов, но и температуры, и плотности различны. Переходим к точной постановке задачи.

Уравнение Больцмана, как и в [16], запишем в виде

$$
\begin{aligned}
\frac{\partial f}{\partial t}+v \frac{\partial f}{\partial x}= & \frac{d^{2}}{2} \int_{R^{3}} d v_{1} \int_{\Sigma} d \alpha\left|\left(v-v_{1}, \alpha\right)\right| \times \\
& \times\left[f\left(t, v^{\prime}, x\right) f\left(t, v_{1}^{\prime}, x\right)-f(t, v, x) f\left(t, v_{1}, x\right)\right]
\end{aligned}
$$

или коротко

$$
D(f)=Q(f, f),
$$

где $v^{\prime}=v-\alpha\left(v-v_{1}, \alpha\right) ; v_{1}^{\prime}=v_{1}+\alpha\left(v-v_{1}, \alpha\right) ; \alpha \in \Sigma ; \Sigma-$ единичная сфрера в $R^{3} ; d-$ диаметр молекул; $v \in R^{3}-$ скорость молекулы.

Будем искать решение уравнения (2) в виде бимодального распределения $f=\varphi M+$ $(1-\varphi) M_{0}$ с различными максвеллианами $M$ и $M_{0}$, а именно:

$$
f(t, v, x)=\varphi(t, x) \rho\left(\frac{\beta}{\pi}\right)^{3 / 2} e^{-\beta v^{2}}+[1-\varphi(t, x)] \rho_{0}\left(\frac{\beta_{0}}{\pi}\right)^{3 / 2} e^{-\beta_{0}\left(v-v_{0}\right)^{2}} .
$$

Здесь $\rho, \rho_{0}$ - плотности газа, $v_{0} \in R^{3}$ - вторая массовая скорость (первую мы для простоты положили равной 0$), \beta=1 / 2 T ; \beta_{0}=1 / 2 T_{0}$ - обратные температуры газа, а функция $\varphi(t, x)$ имеет вид

$$
\varphi(t, x)=\left[1+e^{c\left(x^{1}-\mathcal{D} t\right)}\right]^{-1}
$$

где $c, \mathcal{D}$ - произвольные параметры, $x^{1}$ - первая компонента вектора $x \in R^{3}$ (в (3) и (4) обозначения по сравнению с работой [16] несколько изменены). Очевидно, что при $x^{1} \rightarrow$ $\pm \infty$ или $t \rightarrow \pm \infty$ функция $\varphi(t, x)$ стремится либо к 0, либо к 1 , т.е. распределение $f$ в этих случаях стремится к одному из максвеллианов ( $M$ или $\left.M_{0}\right)$.

Формально (3), (4) напоминает распределение Тамма-Мотт-Смита, однако, как уже отмечалось в [16], разница между ними принципиальна из-за того, что при исследовании ударной волны сразу полагают $v_{0}^{2}=v_{0}^{3}=0$ (это соблюдается и для модификаций 
Холвея, Солвена и др., см. $[1$, с. 416$])$, а в нашей схеме $v_{0}$ произвольно, более того, именно случай, когда $v_{0}^{1}=0, v_{0}^{2} \neq 0, v_{0}^{3} \neq 0$, оказывается наиболее интересным $[16,17]$. Отметим в этой связи еще работу [18], в которой на физическом уровне строгости доказывалось, что распределение Тамма-Мотт-Смита точно удовлетворяет уравнению Больцмана. Более строгие рассмотрения [19] не только опровергли этот вывод, но даже позволили получить жесткие оценки снизу для невязки в $L_{2}$-смысле между $D(f)$ и $Q(f, f)$ (в случае моттсмитовского $f$ ).

Наша задача состоит в исследовании поведения невязки

$$
\Delta=\|D(f)-Q(f, f)\|_{L},
$$

где $f$ взято в виде $(3),(4)$ в зависимости от поведения параметров $\rho, \rho_{0}, \beta, \beta_{0}, v_{0}, c$, $\mathcal{D}$. Легко видеть, что случаи $\rho_{0} \rightarrow 0(\rho \rightarrow 0)$ и $c \rightarrow 0$ тривиальны. Поэтому в работе основное внимание уделено рассмотрению ситуации, когда $\beta, \beta_{0} \rightarrow+\infty$ (низкотемпературный предел) при фиксированных ненулевых значениях остальных параметров.

В разделе 2 проведены необходимые вычисления, связанные с низкотемпературным предельным переходом, результаты которых сформулированы в виде теоремы и следствия.

Раздел 3 посвящен обсуждению как полученных результатов, так и иных возможностей, возникающих в связи с наличием большого числа параметров.

\section{2. ОСНОВНЫЕ РЕЗУЛЬТАТЫ}

Наша цель - выяснить низкотемпературное поведение $\left(\beta, \beta_{0} \rightarrow+\infty\right)$ невязки $\Delta$, определенной равенством (5). Прежде всего вычислим $D(f)$ и $Q(f, f)$ (очевидные выкладки, аналогичные проведенным в работе [16], опустим):

$$
D(f)=-\varphi^{2} e^{c\left(x^{1}-\mathcal{D} t\right)} c\left(v^{1}-\mathcal{D}\right)\left(M-M_{0}\right)
$$

(здесь $v^{1}-$ компонента скорости $v$ вдоль оси $x^{1}$ );

$$
\begin{aligned}
Q(f, f)= & \frac{d^{2}}{2} \varphi(1-\varphi) \int_{R^{3}} d v_{1} \int_{\Sigma} d \alpha\left|\left(v-v_{1}, \alpha\right)\right| \times \\
& \times\left[M\left(v^{\prime}\right) M_{0}\left(v_{1}^{\prime}\right)+M_{0}\left(v^{\prime}\right) M\left(v_{1}^{\prime}\right)-M(v) M_{0}\left(v_{1}\right)-M_{0}(v) M\left(v_{1}\right)\right] .
\end{aligned}
$$

Учитывая, что $\varphi(1-\varphi)=\varphi^{2} e^{c\left(x^{1}-\mathcal{D} t\right)}$, из $(6)$ и $(7)$ получим

$$
\begin{aligned}
D(f)-Q(f, f)= & \varphi^{2} e^{c\left(x^{1}-\mathcal{D} t\right)}\left\{c\left(v^{1}-\mathcal{D}\right)\left(M_{0}-M\right)-\right. \\
& -\frac{d^{2}}{2} \int_{R^{3}} d v_{1} \int_{\Sigma} d \alpha\left|\left(v-v_{1}, \alpha\right)\right|\left[M\left(v^{\prime}\right) M_{0}\left(v_{1}^{\prime}\right)+M_{0}\left(v^{\prime}\right) M\left(v_{1}^{\prime}\right)-\right. \\
& \left.\left.-M(v) M_{0}\left(v_{1}\right)-M_{0}(v) M\left(v_{1}\right)\right]\right\}
\end{aligned}
$$

Поскольку при любых $c$ и $\mathcal{D}$ очевидно, что

$$
\sup _{(x, t) \in R^{3} \times R^{1}} \varphi^{2} e^{c\left(x^{1}-\mathcal{D} t\right)}=\frac{1}{4},
$$


из (5), (8) и (1) имеем

$$
\Delta=\frac{1}{4} \int_{R^{3}} d v\left|c\left(v^{1}-\mathcal{D}\right)\left(M_{0}-M\right)-G\left(M, M_{0}\right)-G\left(M_{0}, M\right)+M \mathcal{L}\left(M_{0}\right)+M_{0} \mathcal{L}(M)\right|,
$$

где введены стандартные обозначения для "прибыточных" $G$ и “затратных" $\mathcal{L}$ частей оператора столкновений $Q(f, f)$. Последние два слагаемых легко вычисляются непосредственно с помощью интегрирования по $\alpha$ и замены $w_{1}=\sqrt{\beta_{0}}\left(v_{1}-v_{0}\right)$ и $w_{1}=\sqrt{\beta} v_{1}$, соответственно:

$$
\begin{aligned}
\mathcal{L}\left(M_{0}\right) & =\frac{d^{2} \rho_{0}}{\sqrt{\pi}} \int_{R^{3}} d w_{1}\left|v-v_{0}-\frac{w_{1}}{\sqrt{\beta_{0}}}\right| e^{-w_{1}^{2}}, \\
\mathcal{L}(M) & =\frac{d^{2} \rho}{\sqrt{\pi}} \int_{R^{3}} d w_{1}\left|v-\frac{w_{1}}{\sqrt{\beta}}\right| e^{-w_{1}^{2}} .
\end{aligned}
$$

Для вычисления $G\left(M, M_{0}\right)$ и $G\left(M_{0}, M\right)$ воспользуемся полученным в работе [20] представлением

$$
\begin{aligned}
G\left(M, M_{0}\right)=G\left(M_{0}, M\right)= & \pi^{2} d^{2}\left[2 R \beta \beta_{0}\right]^{-1} M M_{0} \times \\
& \times\left[\Phi\left(1, \frac{1}{2}, Q+R\right)-\Phi\left(1, \frac{1}{2}, Q-R\right)\right],
\end{aligned}
$$

где $\Phi(a, b, z)$ - вырожденная (конфлюэнтная) гипергеометрическая функция Куммера, а величины $Q$ и $R$ определены следуюшим образом (обозначения [20]):

$$
\begin{aligned}
& Q=\frac{1}{2}\left(\beta v^{2}+\beta_{0}\left(v-v_{0}\right)^{2}\right), \\
& R=\left[\frac{1}{4}\left(\beta v^{2}-\beta_{0}\left(v-v_{0}\right)^{2}\right)^{2}+\beta \beta_{0}\left[v \times\left(v-v_{0}\right)\right]^{2}\right]^{\frac{1}{2}} .
\end{aligned}
$$

Из (12) и (13) легко видеть, что при $v_{0} \neq 0$

$$
Q>0, \quad R \geq 0, \quad Q \geq R,
$$

причем $Q=R$ тогда и только тогда, когда

$$
\left(v-\frac{v_{0}}{2}\right)^{2}=\frac{v_{0}^{2}}{4}
$$

т.е. величина $Q-R$ достигает своего равного нулю минимума в $R^{3}$ на сфере с центром в точке $v_{0} / 2$ и радиусом $\left|v_{0}\right| / 2$. Величина $R$ достигает своего минимума в $R^{3}$ (т.е. обращается в нуль) при

$$
v=\frac{\sqrt{\beta_{0}}}{\sqrt{\beta_{0}} \pm \sqrt{\beta}} v_{0}
$$

Одна из точек (16) не сушествует, если $\beta_{0}=\beta$, другая существует всегда. Поскольку случай $\beta_{0}=\beta$ подробно рассмотрен в $[16,17]$, будем в дальнейшем считать, что $\beta_{0} \neq \beta$.

5 Теоретическая и математическая физика, т. 114, № 1, 1998 г. 
Это удобно формализовать путем введения нового параметра $k$, характеризующего “перепад" температур максвеллианов:

$$
\beta_{0}=k \beta, \quad k>0, \quad k \neq 1 .
$$

Тогда $R$ достигает нулевого значения в двух (различных!) точках (16), причем обе эти точки не лежат на сфере (15). Учитывая $(9),(10),(11)$ и (17), по аналогии с работой [16] оценим невязку $\Delta$ сверху следующим образом:

$$
4 \Delta \leq I_{1}+I_{2}
$$

где

$$
\begin{aligned}
I_{2}= & \rho\left(\frac{\beta}{\pi}\right)^{3 / 2} \int_{R^{3}} d v e^{-\beta v^{2}} \mid c\left(v^{1}-\mathcal{D}\right)+ \\
& +\rho_{0} \frac{\sqrt{k} \sqrt{\pi}}{2 \sqrt{\beta} R} d^{2} e^{-k \beta\left(v-v_{0}\right)^{2}}\left[\Phi\left(1, \frac{1}{2}, Q+R\right)-\Phi\left(1, \frac{1}{2}, Q-R\right)\right]- \\
& -\frac{d^{2} \rho_{0}}{\sqrt{\pi}} \int_{R^{3}} d w_{1}\left|v-v_{0}-\frac{w_{1}}{\sqrt{k \beta}}\right| e^{-w_{1}^{2}} \mid,
\end{aligned}
$$

$I_{1}$ выглядит совершенно аналогично с заменой $v_{0} \leftrightarrow 0, k \beta \leftrightarrow \beta$ и $\rho \leftrightarrow \rho_{0}$.

Используем в дальнейшем для краткости выкладок введенный в [16] символ “эквивалентности при $\beta \rightarrow+\infty$ ": если $J(\beta)$ и $H(\beta)$ - две функции параметра $\beta$, то запись $J \backsim H$ означает, что

$$
\lim _{\beta \rightarrow+\infty}(J-H)=0
$$

(сушествование предела каждой из величин $J$ и $H$ не обязательно). Тогда из (19), применяя технику работы [16], можно получить, что

$$
\begin{aligned}
I_{2} \backsim & \rho\left(\frac{\beta}{\pi}\right)^{3 / 2} \int_{R^{3}} d v e^{-\beta v^{2} \times} \\
& \times\left|B+\rho_{0} \frac{\sqrt{\pi} \sqrt{k}}{2 \sqrt{\beta} R} d^{2} e^{-k \beta\left(v-v_{0}\right)^{2}}\left[\Phi\left(1, \frac{1}{2}, Q+R\right)-\Phi\left(1, \frac{1}{2}, Q-R\right)\right]\right|,
\end{aligned}
$$

где

$$
B=-\mathcal{D} c-\rho_{0} \pi d^{2}\left|v_{0}\right| .
$$

Заметим сразу, что интегралы (19), (21) сходятся (см. приложение). Функция $\Phi(1,1 / 2, z)$ при $z>1$ описывается асимптотической формулой [21]

$$
\Phi\left(1, \frac{1}{2}, z\right)=\sqrt{\pi z} e^{z}+O\left(\frac{1}{z}\right) .
$$

Используя (23), а также определение и свойства величин $Q$ и $R(12)-(16)$, и вновь опираясь на технику работы [16], из (21) получим

$$
I_{2} \backsim \rho\left(\frac{\beta}{\pi}\right)^{3 / 2} \int_{R^{3}} d v e^{-\beta v^{2}}\left|B+\sqrt{Q+R} \rho_{0} \frac{\pi \sqrt{k} d^{2}}{2 \sqrt{\beta} R} \exp \left[Q+R-\beta k\left(v-v_{0}\right)^{2}\right]\right| .
$$


Аналогичное выражение встречается и в [16]. Однако дальнейший анализ по схеме этой работы здесь невозможен вследствие гораздо более сложной структуры величин $Q$ и $R$ при $k \neq 1$. Поэтому предел правой части $(24)$, когда $\beta \rightarrow+\infty$, удается вычислить лишь при некотором предположении. Именно, положим величину $B$ равной нулю, тогда

$$
I_{2} \backsim \rho \rho_{0} \frac{\beta d^{2} \sqrt{k}}{2 \sqrt{\pi}} \int_{R^{3}} d v \exp \left\{-\beta v^{2}-k \beta\left(v-v_{0}\right)^{2}+Q+R\right\} \frac{\sqrt{Q+R}}{R} .
$$

Прежде чем исследовать (25), обсудим предположение $B=0$. На самом деле это требование налагается лишь при анализе $I_{2}$, а рассмотрение $I_{1}$ приводит к аналогичному требованию $E=0$, где

$$
E=c\left(v_{0}^{1}-\mathcal{D}\right)+\rho \pi d^{2}\left|v_{0}\right| .
$$

Легко видеть, что система условий $B=E=0$ совместна и налагает лишь некоторые ограничения на поведение параметров, остаюшихся пока свободными. Интересным, на наш взгляд, является условие совместности

$$
v_{0}^{1}=\mathcal{D}\left(1+\frac{\rho}{\rho_{0}}\right)
$$

из которого следует, что $\left|v_{0}^{1}\right|>|\mathcal{D}|$. Последнее ограничение, видимо, имеет определенный физический смысл, ибо $\mathcal{D}$ - аналог фазовой скорости "волны" (потока), а $v_{0}^{1}$ - соответствуюшая составляющая массовой скорости.

Возврашаясь к исследованию выражения (25), произведем замену

$$
v=u \frac{\left|v_{0}\right|}{2}+\frac{v_{0}}{2} .
$$

Тогда с учетом (12), (13) и (17) получим

$$
I_{2} \backsim \rho \rho_{0} \frac{d^{2} \sqrt{k}}{2 \sqrt{\pi}} \sqrt{\beta}\left(\frac{\left|v_{0}\right|}{2}\right)^{3} \int_{R^{3}} d u P(u, k) e^{-\beta S(u, k)},
$$

где

$$
\begin{aligned}
& P(u, k)=\sqrt{\frac{\beta(Q+R)}{R^{2}}}, \\
& S(u, k)=\frac{1}{2 \beta}(Q-R) v_{0}^{2} .
\end{aligned}
$$

Далее, переходя сначала в (29) к сферической системе координат с осью $z$, направленной вдоль $v_{0}$ (введены обозначения $r=|u|, \pi / 2-\psi$ - угол между векторами $u$ и $v_{0}$ ), а затем вводя переменную $s=\sin \psi$, будем иметь

$$
I_{2} \backsim \rho \rho_{0} \frac{d^{2} \sqrt{k}}{4 \sqrt{2}} \sqrt{\pi \beta} v_{0}^{2} \int_{-1}^{1} d s \int_{0}^{+\infty} P(r, s, k) e^{-\beta S(r, s, k)}=F(\beta, k),
$$


где

$$
\begin{aligned}
P(r, s, k) & =r^{2} \sqrt{\frac{\frac{1}{\beta} Q(r, s, k)+\frac{1}{\beta} R(r, s, k)}{\left(\frac{1}{\beta} R(r, s, k)\right)^{2}}}, \\
S(r, s, k) & =\left(\frac{1}{\beta} Q(r, s, k)-\frac{1}{\beta} R(r, s, k)\right) \frac{v_{0}^{2}}{2} \\
\frac{1}{\beta} Q(r, s, k) & =\frac{1+k}{4} r^{2}+\frac{1}{2} r s(1-k)+\frac{1}{4}(1+k), \\
\frac{1}{\beta} R(r, s, k) & =\left[\left(\frac{1-k}{4} r^{2}+\frac{1}{2} r s(1+k)+\frac{1-k}{4}\right)^{2}+k r^{2}\left(1-s^{2}\right)\right]^{\frac{1}{2}} .
\end{aligned}
$$

Итак, нас интересует асимптотика при $\beta \rightarrow+\infty$ двойного интеграла $F(\beta, k)$, взятого по полуполосе:

$$
\Omega=\left\{(r, s) \in R^{2}: r \geq 0 ;-1 \leq s \leq 1\right\} .
$$

Имея целью применить здесь метод Лапласа, перечислим основные свойства функций $P$ и $S$ (они легко проверяются исходя из (33)-(36)):

$$
\max _{\Omega}\{-S(r, s, k)\}=0=S(1, s, k),
$$

т.е. глобальный максимум функции $-S$ на $\Omega$ достигается на отрезке $A=\left\{(r, s) \in R^{2}\right.$ : $r=1 ;-1 \leq s \leq 1\}$; функция $P(r, s, k)$ бесконечно дифференцируема в окрестности отрезка $A$ и непрерывна всюду в $\Omega$, кроме двух интегрируемых особенностей, не лежаших на $A$, так что интеграл (32) абсолютно сходится; кроме того,

$$
P(r, s, k)>0, \quad(r, s) \in \Omega,
$$

и наконец,

$$
-S_{r r}^{\prime \prime}(1, s, k)=\frac{-k v_{0}^{2}}{(s+1)+k(1-s)}<0 .
$$

Значит, к интегралу (32) может быть применена теорема Федорюка [22, гл. II, теорема 4.8$]$, которая дает

$$
F(\beta, k)=\rho \rho_{0} \frac{d^{2} \sqrt{k}}{4 \sqrt{2}} \pi v_{0}^{2}\left[\int_{A} P(r, s, k) \omega_{h}+o(1)\right],
$$

где $o(1) \rightarrow 0$ при $\beta \rightarrow+\infty$; криволинейный интеграл берется по отрезку $A$, a $\omega_{h}-$ дифоференциальная форма Лере-Гельфанда для функции $h$ :

$$
h(r, s, k)=\sqrt{-S(r, s, k)} .
$$

Из (41) и (42) прямым вычислением получаем

$$
I_{2} \backsim \rho \rho_{0} \pi d^{2}\left|v_{0}\right| .
$$


Сразу же заметим, что результат не зависит от $k$, т.е. при любом фиксированном перепаде температур в исходном бимодальном распределении низкотемпературный предел величины $I_{2}$ один и тот же! Аналогичное, разумеется, верно и для $I_{1}$. Более того, опираясь на другую теорему [22, гл. II, теорема 2.1] и рассматривая переменные $s, k$ как двумерный параметр в одномерном интеграле по $r$, нетрудно показать, что $F(\beta, k)$ стремится к пределу (43) при $\beta \rightarrow+\infty$ равномерно на любом отрезке $k \in\left[k_{1}, k_{2}\right]$, где $k_{1}, k_{2}>0$ (ограничение $k \neq 1$ также снимается). Наконец, очевидно, что результат (43) при $\rho=\rho_{0}=1$ соответствует результату работы [16].

Все вьшеизложенное позволяет считать доказанной следуюшую теорему.

Теорема. Если параметры бимодального распределения (3), (4) связаны соотношениями

$$
\begin{gathered}
B=-\mathcal{D} c-\rho_{0} \pi d^{2}\left|v_{0}\right|=0, \quad E=c\left(v_{0}^{1}-\mathcal{D}\right)+\rho \pi d^{2}\left|v_{0}\right|=0, \\
\beta_{0}=k \beta, \quad 0<k<+\infty
\end{gathered}
$$

mo

$$
\lim _{\beta \rightarrow+\infty} I_{1}=\lim _{\beta \rightarrow+\infty} I_{2}=\rho \rho_{0} \pi d^{2}\left|v_{0}\right|,
$$

где $I_{1}$ и $I_{2}$ определены в (18), (19).

Исходя из (18) и выражений для $B$ и $E$ легко видеть, что для "околосвободномолекулярного" течения ( $d$ мало) имеет место следуюшее следствие из этой теоремы.

СлЕДСтвиЕ. Пусть величинь $v_{0}^{2} \neq 0, \quad v_{0}^{3} \neq 0, \quad c \neq 0, \quad \rho>0, \quad \rho_{0}>0, \quad k>0$ произвольны и фиксировань. Тогда для каждого $\epsilon>0$ найдется такое $\delta>0$, что для любых $\mathcal{D}, v_{0}^{1}, d$, связанных соотношениями $B=E=0$ и удовлетворяющих условиям $|\mathcal{D}|<\delta, \quad\left|v_{0}^{1}\right|<\delta, \quad 0<d<\delta$, существует такое $N>0$, что при всех $\beta>N$ справедливо неравенство

$$
\Delta=\|D(f)-Q(f, f)\|_{L}<\epsilon .
$$

Этот факт проше всего можно записать с помошью специального "повторного" предельного перехода

$$
\lim _{\substack{\mathcal{D} \rightarrow 0 \\ v_{0}^{1} \rightarrow 0 \\ d \rightarrow 0}} \lim _{\substack{\mathrm{d} \\ d \rightarrow+\infty}} \Delta=0,
$$

где внешний предел берется по "траектории" в пространстве параметров $\mathcal{D}, v_{0}^{1}, d$, определяемой условиями $B=E=0$. Разумеется, (45) следует понимать лишь символически, т.к. сушествование внутреннего предела при $k \neq 1$ на самом деле доказано не для самого $\Delta$, а лишь для величин $I_{1}$ и $I_{2}$.

ЗАмЕчаниЕ. Легко видеть, что при $B=E=0$ стремление любого из параметров $\mathcal{D}, v_{0}^{1}, d$ к нулю влечет за собой стремление к нулю и двух других. 


\section{3. ОБСУЖДЕНИЕ РЕЗУЛЬТАТОВ}

Доказанные теорема и следствие аналогичны результатам работы [16], т.е. случай различных температур принципиально не отличается от случая равных температур. Технические трудности не позволяют перенести на двухпотоковые решения результаты работы [17] и избавиться от требований $B=E=0$. Тем не менее можно считать установленным, что при больших числах Кнудсена возможны течения газа из твердых сфер, сколь угодно близкие к двухпотоковому, причем перепад температур $k$ произволен. Помимо предельного перехода (45), имеется много других возможностей, связанных с остальными параметрами распределения $f$. Отметим некоторые из них (другие либо затруднительны для исследования, либо тривиальны: например, при $c \rightarrow 0$ исчезает зависимость от $x$ и $t$, при $d \rightarrow 0$ сразу получаем кнудсеновский газ и т.п.).

1. Интересно было бы в (25) сначала устремить $k$ к нулю или бесконечности при фиксированном $\beta$. Однако зависимость подынтегральной функции от параметра $k$ весьма сложна, и не удается строго обосновать предельный переход по $k$. Если же осушествить его формально, то полученная функшия от $\beta$ в пределе $\beta \rightarrow+\infty$ все равно дает (43).

2. При $k \neq 1$ приобретает интерес и случай $v_{0} \rightarrow 0$ (или даже $v_{0}=0$ ), который тривиален при $k=1$. Легко видеть, что тогда для любого $d>0$

$$
\lim _{\mathcal{D} \rightarrow 0} \lim _{v_{0} \rightarrow 0} \lim _{\beta, \beta_{0} \rightarrow+\infty} \Delta=\lim _{\mathcal{D} \rightarrow 0} \lim _{\beta, \beta_{0} \rightarrow+\infty} \lim _{v_{0} \rightarrow 0} \Delta=0 .
$$

Сушественно, что в этом случае $d$ произвольно, т.е. (46) имеет место для любого газа, а не только близкого к кнудсеновскому. Правда, физический смысл этой ситуации не столь интересен, ибо здесь и $M$, и $M_{0}$ стремятся к $\delta$-функции, сосредоточенной в нуле (хотя и с разной скоростью). Это значит, что в пределе мы получаем "мертвый газ", т.е. все молекулы покоятся.

3. Интересный эффект наблюдается при попытке поменять местами два предельных перехода $\beta \rightarrow+\infty$ и $v_{0} \rightarrow 0$ в случае $k=1, \rho=\rho_{0}=1$ (остальные параметры фиксированы). Тогда очевидно, что

$$
\lim _{\beta \rightarrow+\infty} \lim _{v_{0} \rightarrow 0} \Delta=0
$$

(т.к. $\lim _{v_{0} \rightarrow 0} \Delta\left(\beta, v_{0}\right)=\Delta(\beta, 0)=0$, поскольку при указанных выше условиях и $v_{0}=0$ распределение $f$ совпадает с максвеллианом). С другой стороны, из результатов $[17]$ вытекает, что

$$
\lim _{v_{0} \rightarrow 0} \lim _{\beta \rightarrow+\infty} \Delta=\frac{1}{2}|\mathcal{D} c| .
$$

Мы видим, что (47) и (48) не совпадают, т.е. функция

$$
\widetilde{\Delta}\left(T, v_{0}\right)=\Delta\left(\frac{1}{2 T}, v_{0}\right)=\Delta\left(\beta, v_{0}\right)
$$

не является непрерывной в точке $T=0, v_{0}=0$.

4. Как отмечалось в $[16,17]$, уже на первом этапе предельного перехода (45), т.е. при $\beta \rightarrow+\infty$, функция $f$, а также $D(f)$ и $Q(f, f)$ выходят из пространства $L$ и имеют предел лишь в пространстве обобшенных функций, однако величина $\Delta$, т.е. норма разности 
$D(f)-Q(f, f)$ в пространстве $L$, при $k=1$ имеет конечный предел (по-видимому, это будет так и при $k \neq 1$, хотя строгого доказательства пока нет). Значит, можно сказать, что уравнение Больцмана для функций $f$ рассматриваемого вида ведет себя более регулярным образом, чем сами функции $f$.

Автор выражает искреннюю признательность А. В. Бобылеву за полезные обсуждения полученных результатов и ценные замечания.

\section{ПРИЛОЖЕНИЕ}

Докажем, например, сходимость интеграла (21). Подынтегральная функция с точностью до констант не превосходит суммы

$$
\begin{gathered}
|B| e^{-\beta v^{2}}+\frac{1}{R} \exp \left\{-\beta v^{2}-k \beta\left(v-v_{0}\right)^{2}\right\} \Phi\left(1, \frac{1}{2}, Q+R\right)+ \\
+\frac{1}{R} \exp \left\{-\beta v^{2}-k \beta\left(v-v_{0}\right)^{2}\right\} \Phi\left(1, \frac{1}{2}, Q-R\right) .
\end{gathered}
$$

Интеграл от первого слагаемого в (49) сходится, а третье из них, очевидно, мажорируется вторым. В силу асимптотики (23) достаточно таким образом обосновать сходимость интеграла

$$
I=\int_{R^{3}} d v \exp \left\{-\beta v^{2}-k \beta\left(v-v_{0}\right)^{2}+Q+R\right\} \frac{\sqrt{Q+R}}{R} .
$$

Благодаря (12) и (17) интеграл (50) упрошается, и мы имеем

$$
I=\int_{R^{3}} d v e^{-(Q-R)} \frac{\sqrt{Q+R}}{R} .
$$

Приведем величины $R$ и $Q-R$ к более удобному виду исходя из $(12),(13)(\theta-$ угол между векторами $v$ и $\left.v-v_{0}\right)$ :

$$
\begin{aligned}
R & =\left[\frac{1}{4}\left(\beta v^{2}-\beta_{0}\left(v-v_{0}\right)^{2}\right)^{2}+\beta \beta_{0} v^{2}\left(v-v_{0}\right)^{2} \sin ^{2} \theta\right]^{1 / 2}= \\
& =\left[\frac{1}{4}\left(\beta v^{2}+\beta_{0}\left(v-v_{0}\right)^{2}\right)^{2}-\beta \beta_{0} v^{2}\left(v-v_{0}\right)^{2} \cos ^{2} \theta\right]^{1 / 2}= \\
& =\left[\frac{1}{4}\left(\beta v^{2}+\beta_{0}\left(v-v_{0}\right)^{2}\right)^{2}-\beta \beta_{0}\left(v, v-v_{0}\right)^{2}\right]^{1 / 2}= \\
& =\frac{1}{2}\left|\sqrt{\beta} v-\sqrt{\beta_{0}}\left(v-v_{0}\right)\right|\left|\sqrt{\beta} v+\sqrt{\beta_{0}}\left(v-v_{0}\right)\right|= \\
& =\frac{1}{2}\left|v\left(\sqrt{\beta_{0}}-\sqrt{\beta}\right)-\sqrt{\beta_{0}} v_{0}\right|\left|v\left(\sqrt{\beta_{0}}+\sqrt{\beta}\right)-\sqrt{\beta_{0}} v_{0}\right| .
\end{aligned}
$$

Во-первых, из (52) видно, что величина $R$ имеет в точках (16) корни первого порядка, 
что приводит к двум интегрируемым полюсам в (51). Во-вторых, используя (52), получим

$$
\begin{aligned}
Q-R= & \frac{1}{2}\left(\beta v^{2}+\beta_{0}\left(v-v_{0}\right)^{2}\right)-\frac{1}{2}\left|\sqrt{\beta} v-\sqrt{\beta_{0}}\left(v-v_{0}\right)\right|\left|\sqrt{\beta} v+\sqrt{\beta_{0}}\left(v-v_{0}\right)\right|= \\
= & \frac{1}{4}\left[\left(\sqrt{\beta} v-\sqrt{\beta_{0}}\left(v-v_{0}\right)\right)^{2}+\left(\sqrt{\beta} v+\sqrt{\beta_{0}}\left(v-v_{0}\right)\right)^{2}-\right. \\
& \left.-2\left|\sqrt{\beta} v-\sqrt{\beta_{0}}\left(v-v_{0}\right)\right|\left|\sqrt{\beta} v+\sqrt{\beta_{0}}\left(v-v_{0}\right)\right|\right]= \\
= & \frac{1}{4}\left(\left|\sqrt{\beta} v+\sqrt{\beta_{0}}\left(v-v_{0}\right)\right|-\left|\sqrt{\beta} v-\sqrt{\beta_{0}}\left(v-v_{0}\right)\right|\right)^{2}= \\
= & \frac{1}{4}\left(\left|v\left(\sqrt{\beta_{0}}+\sqrt{\beta}\right)-\sqrt{\beta_{0}} v_{0}\right|-\left|v\left(\sqrt{\beta_{0}}-\sqrt{\beta}\right)-\sqrt{\beta_{0}} v_{0}\right|\right)^{2} .
\end{aligned}
$$

При $v \rightarrow \infty$ выражение (53), очевидно, имеет следующий асимптотический вид:

$$
\frac{1}{4} v^{2}\left(\sqrt{\beta_{0}}+\sqrt{\beta}-\left|\sqrt{\beta_{0}}-\sqrt{\beta}\right|\right)^{2},
$$

т.е. на бесконечности интеграл (51) ведет себя одинаково с интегралом

$$
\int_{R^{3}} d v \exp \left\{-\frac{1}{4} v^{2}\left(\sqrt{\beta_{0}}+\sqrt{\beta}-\left|\sqrt{\beta_{0}}-\sqrt{\beta}\right|\right)^{2}\right\} \sqrt{\frac{Q+R}{R^{2}}} .
$$

Коэффициент при $v^{2}$ в экспоненте отрицателен, а подкоренное выражение, как видно из $(12)$ и $(52)$, ограничено при $v \rightarrow \infty$. Следовательно, интеграл (55) сходится, что и требовалось.

\section{Список литературы}

[1] K. Черчиньяни. Теория и приложения уравнения Больцмана. М.: Мир, 1978.

[2] Т. Карлеман. Математические задачи кинетической теории газов. М.: ИИЛ, 1960.

[3] R.S. Krupp. Magister Sciences thesis: MiT 1967.

[4] А. В. Бобылев. Докл. АН СССР. 1975. Т. 225. № 6. С. 1296-1299

[5] А. В. Бобылев. Докл. АН СССР. 1976. Т. 231. № 3. С. 571-574.

[6] M. Krook, T. Wu. Phys. Fluids. 1977. V. 20. № 10(1). Р. 1589-1595.

[7] А. А. Никольский. Докл. АН СССР. 1963. Т. 151. № 2. С. 299-302.

[8] E. Ikenberry, C. Truesdall. Ration Mech. and Anal. 1956. V. 5. № 1. P. 1-129.

[9] В. С. Галкин. Прикл. матем. и мех. 1956. Т. 20. № 3. С. 445-446.

[10] H. M. Ernst. J. Stat. Phys. 1984. V. 34. № 5/6. P. 1001-1017.

[11] А. В. Бобылев. Докл. АН СССР. 1980. Т. 251. № 6. С. 1361-1365.

[12] В. В. Веденяпин. Докл. АН СССР. 1981. Т. 256. № 2. С. 338-342.

[13] Д. Я. Петрина, А. В. Мищенко. Докл. АН СССР. 1988. Т. 298. № 2. С. 338-342.

[14] А. В. Мищенко, Д. Я. Петрина. ТМФ. 1988. Т. 77. № 1. С. 135-153.

[15] И. Е. Тамм. Труды ФИАН. 1965. Т. 29. С. 239-249.

[16] В. Д. Гордевский. Матем. физ., анализ, геом. 1995. Т. 2. № 2. С. 168-176.

[17] В. Д. Гордевский. Матем. физ., анализ, геом. 1997. Т. 4. № 1/2. С. 1-12.

[18] A. Sakurai. J. Fluid Mech. 1957. Part 3. P. 255-260.

[19] R. Narasimha, S. M. Deshpande. J. Fluid Mech. 1969. V. 36. № 3. P. 555-570.

[20] S. M. Deshpande, R. Narasimha. J.Fluid Mech. 1969. V. 36. № 3. P. 545-554.

[21] Е. Янке, Ф. Эмде, Ф. Леш. Специальные функции. М.: Наука, 1964.

[22] М. Ф. Федорюк. Метод перевала. М.: Наука, 1977. 